Med Care. 2009 June ; 47(6): 610-617. doi:10.1097/MLR.0b013e31819748d5.

\title{
The Payoff Time:
}

\section{A Flexible Framework to Help Clinicians Decide When Patients With Comorbid Disease are not Likely to Benefit From Practice Guidelines}

\author{
R. Scott Braithwaite, MD, MSc, David Fiellin, MD, and Amy C. Justice, MD, PhD \\ Department of Internal Medicine, Section of General Internal Medicine, Yale University School of \\ Medicine and VA Connecticut Healthcare System, New Haven, Connecticut.
}

\section{Abstract}

Background-Practice guidelines rarely consider comorbid illness, and resulting overuse of health services may increase costs without conferring benefit.

Objective-To individualize a framework for inferring when patients with comorbid illness are not likely to benefit from colorectal cancer screening guidelines.

Methods-We modified the "payoff time" framework (the minimum time until a guideline's cumulative benefits exceed its cumulative harms) to increase its applicability to a wide range of primary care patients. We show how it may inform colorectal (CR) cancer screening decisions for 3 typical patients in general practice for whom CR screening would be recommended by current guidelines: (1) 60-year-old man with diabetes, congestive heart failure, lung disease, stroke, and substantial frailty; (2) 60-year-old woman with diabetes and obesity, without other comorbidity or frailty; and (3) 50-year-old woman with inflammatory bowel disease.

Results-For patient 1, the payoff time for CR screening (minimum time until benefits exceed harms) is 7.3 years, and for patient 2, the payoff time for CR screening is 5.4 years. Evidence is insufficient to estimate the payoff time for patient 3 . Because patient 1's estimated life expectancy is 3.7 years (less than his payoff time), he is unlikely to benefit from CR screening. Because patient 2's estimated life expectancy exceeds 10 years (greater than her payoff time), she may benefit from CR screening. Because evidence is insufficient to estimate the payoff time for patient 3 , the payoff time framework does not inform decision making.

Conclusion-The payoff time framework may identify patients for whom particular clinical guidelines are unlikely to confer benefit, and has the potential to decrease unnecessary health care.

Patients with chronic illness may not survive long enough to gain from practice guidelines that have immediate risks and delayed benefits. $1^{-} 3$ For example, chronically ill patients receiving screening colonoscopy incur the risk of colonoscopy-related harms (eg, perforation, substantial bleeding) but may not live long enough to benefit from lowered colorectal cancer risk. Chronically ill persons receiving screening abdominal ultrasounds incur the risk of surgery-related complications if an aneurysm is detected, but may not live long enough to benefit from decreased risk of spontaneous aneurysm rupture. While clinicians have the option of withholding practice guidelines when harm is likely to exceed benefit, a substantial body of evidence suggests that clinicians do not make this assessment accurately, and chronically ill individuals receive interventions that may be harmful.4,5

Copyright $@ 2009$ by Lippincott Williams \& Wilkins

Reprints: R. Scott Braithwaite, MD, MSc, Department of Internal Medicine, Section of General Internal Medicine, Yale University School of Medicine and VA Connecticut Healthcare System, New Haven, CT. Ronald.Braithwaite@va.gov. 
Furthermore, the advent of incentives and contingencies (eg, pay for performance, quality "benchmarks") may reinforce inclinations to apply practice guidelines rigidly, despite chronic diseases. 6

We have previously developed a framework termed the "payoff time." The payoff time is designed to identify the minimum elapsed time until the cumulative incremental benefits attributable to a practice guideline first exceed the cumulative incremental harms associated with applying the guideline.7 A long payoff time means that it takes an extended time until benefits outweigh harms, whereby a short payoff time means that benefits rapidly outweigh harms. The payoff time is relevant to any guideline that involves immediate harms and delayed benefits, and, in theory, can be used to predict whether a particular clinical guideline should apply to a particular patient if his life expectancy can be estimated. If the payoff time is greater than that patient's life expectancy (ie, as may happen for chronically ill patients with low life expectancies) then the guideline should not apply because the patient is unlikely to live long enough to accrue more benefit than harm. On the other hand, if the payoff time is less than that patient's life expectancy (ie, as may happen for healthy patients with greater life expectancies) then the guideline may apply, because the patient is likely to live long enough to accrue more benefit than harm.

While the payoff time has promise for informing care decisions, it may be difficult to apply in practice because; (1) it requires prognostic information that is specific to comorbid disease(s) that is often not available to the clinician "at the bedside," (2) does not consider generic predictors of mortality such as frailty (ie, the correlates and sequelae of decreased functional reserve), (3) does not consider interactions between comorbidities, frailty, and diseases targeted by practice guidelines, and 4) does not consider patient preferences. In this report, we describe how the payoff time approach can be modified to address these limitations, and thereby may be used in "real world" settings to reduce inappropriate utilization. We use it to inform colorectal cancer screening decisions for 3 typical clinic patients in whom colorectal cancer screening would be recommended by current practice guidelines1: 60-year-old man with diabetes, congestive heart failure, lung disease, stroke, and substantial frailty2; 60-year-old woman with diabetes and obesity, without other comorbidity or frailty; and3 50-year-old woman with inflammatory bowel disease.

\section{METHODS}

We first describe how we modified the payoff time framework to make it more useful to the clinician, and then how we can use it to inform decisions about who to screen for colorectal cancer.

\section{Modifying the Payoff Time Framework}

Making the Framework More Generic-Applying the payoff time requires estimating life expectancy. The previous description of the payoff time framework suggested using disease-specific prognostic models (eg, survival in congestive heart failure patients) to estimate life expectancies.7 However, this approach restricts the spectrum of patients to which the framework can be applied, because validated prognostic models for patients with diseases that are comorbid (eg, survival in patients with congestive heart failure and diabetes) are often not available. Furthermore, different prognostic models are needed for different types of patients, complicating its application. For these reasons, we have modified the payoff time framework to incorporate estimates from a prognostic model that was developed in patients who had a variety of comorbid conditions. 8 We chose to use this framework because it has good discrimination (c statistic of 0.84 in the development cohort and 0.82 in the validation cohort), was developed on a sample generalizable to primary care 
(a population-based sample of community dwelling US adults older than 50 years), and contains a limited number of items (13) that can be easily administered in the office.

To adapt the prognostic model's estimates of 4-year survival to the payoff time's estimates of life expectancy we used the declining exponential approximation of life expectancy (DEALE). The DEALE is a simple approach that allows one to convert data from various literature sources (eg, 4-year survival) into life expectancy.9 The DEALE has not been regularly used as a clinical tool because it is only valid to apply when annual mortality is $\geq 10 \%$.9 However, because the payoff time framework generally will not influence care when annual mortality is less than $10 \%$ (the corresponding life expectancies will exceed 10 years, longer than most payoff times), this limitation of the DEALE does not restrict our approach.

\section{Incorporating Frailty}

Frailty is a biologic construct that is characterized by declining functional reserve, and confers vulnerability to disability, hospitalization, nursing facility admission, and death beyond the impact of age and known medical conditions.10,11 For example, a thin, fragile, homebound 80-year-old woman may have osteoporosis as her only known comorbidity, yet her frailty places her at far higher risk for death than would be expected based on her age and osteoporosis alone. We expanded the payoff time framework because frailty may frequently cause life expectancy to fall to below a particular payoff time (Fig. 1).

Measurements of frailty usually include signs of decreased functional reserve such as slowness, weakness, exhaustion, and wasting10; or consequences such as falls, disability, and institutionalization.12,13 The prognostic model of Lee et al evaluated a large number of frailty items for predictive value, including difficulties with activities of daily living and instrumental activities of daily living, and the final model includes a substantial proportion of items that may arise from frailty (wasting, difficulty bathing, difficulty managing money, difficulty walking several blocks, and difficulty pulling or pushing large objects). Although there are additional frailty markers that were not evaluated or included in the model (eg, falls, hospitalizations), that may conceivably add precision to life expectancy estimation, we reasoned that the potential advantages of including these predictors in our framework were outweighed by the disadvantage of modifying a validated instrument. Consequently, we believe that the model of Lee et al was sufficient to consider frailty in our framework.

\section{Considering Interactions Between Comorbidities, Frailty, and Diseases Targeted by Practice Guidelines}

Just as life expectancy may be influenced by comorbidity and frailty, the payoff time may also be influenced by these factors. For example, the payoff time for colorectal cancer screening via colonoscopy for average-risk 60-year-old men (5.4 years) may be expected to be longer for patients with characteristics that increase the likelihood of harm from screening more than the likelihood of benefit (eg, frailty, which would make complications from colonoscopy more likely and/or more severe). Conversely, the payoff time may be expected to be shorter for patients with characteristics that increase the likelihood of benefit from screening more than the likelihood of harm (eg, major risk factors for colon cancer). Consequently, we reasoned that the payoff time framework can consider interactions if it is applied in accord with the following decision rules (Fig. 2).

Rule A: If the impact of comorbidities and/or frailty on guideline-associated harms and benefits is not likely to be substantial (ie, a statistically and clinically significant effect on an important harm or benefit, based on high-quality evidence), then the payoff time can be used without adjustment. 
However, in many clinical situations the expected impact of comorbidities and/or frailty on guideline-associated harms and benefits is expected to be substantial. In this situation, other decision rules may need to be invoked.

Rule B: If the impact of comorbidities and/or frailty on guideline-associated harms and benefits is substantial and can be quantified, then the payoff time should be adjusted to reflect these harms and benefits before it is used.

However, often the impact of comorbidity and/or frailty will be difficult to quantify, and the final decision rule may need to be invoked.

Rule C: If the impact of comorbidities and/or frailty on guideline-associated harms and benefits is likely to be substantial but is unknown, then the payoff time should not be used if the impact on benefits is likely to be underestimated, or if the impact on harms is likely to be overestimated. Otherwise, it is reasonable to use the payoff time without adjustment.

The reasoning behind this rule is as follows. If there is no underestimation of benefits or overestimation of harms, then the estimated payoff time will be lesser than or equal to the true payoff time. Consequently, if a patient's life expectancy is below an underestimated payoff time, it would also be below the true payoff time, so it would remain valid to use the estimated payoff time to infer that a guideline may harm that patient. However, if there is overestimation of benefits or underestimation of harms, the estimated payoff time may be greater than the true payoff time. Then, a patient's life expectancy could be below the estimated payoff but still could be above the true payoff time, so it would be invalid to use the estimated payoff time to infer that a guideline may harm that patient. It is preferable to avoid using the payoff time framework altogether in this situation.

We used previously published methods to estimate the mortality payoff time for colorectal cancer screening 7 with 2 modifications. First, we updated our estimate of the incidence of serious complications after colonoscopy by $25 \%$ (from 2.0 per 1000 to 2.5 per 1000) based on 2 recently published studies of adverse events following colonoscopies performed at large integrated health delivery systems (incidence of perforation, bleeding requiring transfusion or hospitalization, diverticulitis requiring surgery or hospitalization, or postpolypectomy syndrome; 2.4 per 1000, Ko et al14; 2.5 per 1000, Levin et al15). Correspondingly, the attributable mortality (ie, chance of death from colonoscopy complications) was revised $25 \%$ upwards (now assumed to be 2.5 per 10,000 rather than 2.0 per 10,000 as in the previous report). Second, our payoff time framework now considers the possibility that the onset of benefits may be delayed. In the current study, the benefit from screening was assumed to begin only after a 5-year delay following screening, in accordance with published randomized controlled trials, 16 rather than instantaneously at the time of screening, as in the previous report. The magnitude of benefit from screening, after this delay, was assumed to be unchanged from our prior report (reducing colorectal cancer mortality by a relative risk of 0.70 ).

\section{Using the Payoff Time to Inform Clinical Decisions}

Using the payoff time to inform clinical decisions requires first estimating the life expectancy for a particular patient, then estimating the payoff time for a particular guideline, adjusting that payoff time if necessary (based on comorbidities, frailty, and other factors that influence the balance of benefits to harms), and then comparing the guideline's payoff time to the patient's life expectancy (Figure 3). The prognostic index of Lee et al (Table 1) can be used to estimate 4 -year probability of death. If it is greater than $33 \%$, then it is likely that life expectancy will be below 10 years (within the range of probable payoff times) and that the DEALE can use it to approximate life expectancy. For colorectal cancer screening, there are 
18 patient characteristics that may require adjustment of the payoff time for an individual patient, or may prevent it from being applicable (Table 2). These characteristics were selected because published reports suggested that their effects were clinically and statistically significant, and (if multiple studies were performed) were consistent in magnitude and direction. Based on these effects, the individualized risk for benefit (based on the individual risk of colorectal cancer death; second column of Table 2) can be divided by the individualized risk for harm (based on the individual risk of death from colonoscopyrelated complications; third column of Table 2). This yields an individualized risk-to-benefit adjustor (first column of Table 3), which tailors the payoff time based on individual patient characteristics (other columns, Table 3 ) in accordance with methods previously published.7 After estimating a guideline's payoff time and adjusting it if necessary, it can be compared with that patient's estimated life expectancy. If the life expectancy is less than the payoff time, the clinician can initiate a shared discussion of how screening is less likely to result in benefit and may produce harm.

The sequence of gathering necessary information to use this approach can be adapted to suit clinic workflow and capabilities of the medical record system, and does not need to reflect the order in which it is used. For the particular guideline under consideration (colorectal cancer screening), even if no electronic medical record (EMR) is available, all necessary information (corresponding to the rows in Tables 1 and 2) could be gathered by using a survey at the time of check-in. If an EMR is available with the capability to search for relevant diagnoses and characteristics, the survey could be shortened to as few as 8 questions.

\section{RESULTS}

We now describe how the payoff time can be applied to inform colorectal cancer screening decisions for 3 typical primary care patients.

\section{Mr. Z, a 60-Year-Old Male With Extensive Comorbidity and Frailty}

Our first case is Mr. Z, a 60-year-old man with diabetes, heart failure, lung disease, previous stroke, and substantial frailty; as evidenced by wasting, difficulty walking several blocks, difficulty pushing or pulling heavy objects, difficulty showering, and difficulty managing money. His medications include aspirin, which reduces the risk for colorectal cancer. We first ask whether the payoff time for colorectal cancer screening is applicable to Mr. Z, and if it needs adjustment (Table 2). Mr. Z has several characteristics that require adjustment of the payoff time (diabetes, aspirin, American Society of Anesthesiology Class 3 ["severe systemic disease"] status), and none of the characteristics that prevent the payoff time from being applicable (history of inflammatory bowel disease, colon cancer or adenomatous polyps, pelvic radiation, Hodgkin lymphoma, and acromegaly). Therefore, Mr. Z's relative risk for colorectal cancer is 1.04 times that of an average-risk person (1.3 from diabetes multiplied by 0.8 from regular aspirin use), and Mr. Z's relative risk for colonoscopy complications is 4.3 times that of an averagerisk person (because he is American Anesthesiology Class 3). Consequently, Mr. Z's benefit to harm adjustor is 1.04 divided by 4.3 or 0.24 . From Table 3 , we see that the payoff time for colorectal cancer screening for a 60 -year-old man with an individualized benefit-to-harm adjustor of 0.24 is slightly under 7.3 years.

Next, we estimate Mr. Z's 4-year probability of death based on the prognostic index of Lee et al (Table 1). Mr. $\mathrm{Z}$ is aged 60 (0 points), male ( 2 points), has wasting that lowers his BMI below 25 (1 point), has diabetes (1 point), congestive heart failure ( 2 points), chronic lung disease ( 2 points), difficulty with bathing ( 2 points), difficulty walking several blocks ( 2 points), difficulty pulling or pushing heavy objects (1 point), and difficulty managing money 
(1 point). In total, Mr. $Z$ has 14 points, which gives him a 4-year probability of death of $66 \%$, and a corresponding life expectancy of 3.7 years. Because the payoff time (7.3 years) exceeds Mr. Z's life expectancy (3.7 years), he is unlikely to benefit from colorectal cancer screening. Accordingly, Mr. Z's clinician should engage him in an informed discussion about how the likely harms exceed the likely benefits.

\section{Mrs. J, a 60-Year-Old Woman With Limited Comorbidity and Without Frailty}

Our second case is Mrs. J, a 60-year-old nurse with obesity and diabetes and no other comorbidities or frailty. She does not take aspirin regularly, even though this was advised. We first ask whether the payoff time for colorectal cancer screening is applicable to this particular patient, and if it needs adjustment. Mrs. J has 2 characteristics that require adjustment of the payoff time (diabetes and obesity) and none of the characteristics that prevent the payoff time from being applicable. Mrs. J's relative risk for colorectal cancer is 1.95 times that of an average-risk person ( 1.5 from obesity, multiplied by 1.3 from diabetes), and Mrs. J's relative risk for colonoscopy complications is the same of that of an averagerisk person because she does not have any characteristics that require modification (Table 3). Therefore, Mrs. J's benefit to harm adjustor is 1.95 divided by 1 or 1.95. Interpolating from Table 3, we see that the payoff time for colorectal cancer screening for a 60 -year-old woman with an individualized benefit-to-harm adjustor of 1.95 is slightly over 5.4 years.

Next, we estimate Mrs. J's 4-year probability of death based on the prognostic index of Lee et al (Table 1). Mrs. J is aged 60 (1 points) and has diabetes (1 point) (giving her only 2 points) for a corresponding mortality risk of $2 \%$. Because this is lower than 33\%, Mrs. J has a life expectancy that is well above 10 years, and therefore above her payoff time for colorectal cancer screening of 5.4 years. Therefore, the benefits of screening will likely accrue to Mrs. J.

\section{Mrs. Q, a 50-Year-Old Woman at High Risk for Colorectal Cancer}

Our third case is Ms. Q, a 50-year-old woman with inflammatory bowel disease, and no other comorbidities or frailty. As before, our first step is to ask whether the payoff time for colorectal cancer screening is applicable to this particular patient, and if it needs adjustment. Because Ms. Q has inflammatory bowel disease, the payoff time may underestimate benefits (Table 2), and therefore we should not use the payoff time framework for this particular patient.

\section{DISCUSSION}

We demonstrate how the payoff time framework can be adapted to inform real world decisions at the point of care and has the potential to reduce unnecessary health care utilization. While this framework is still maturing, the current report shows that it can be modified to use generic prognostic models, include frailty, and to consider interactions between comorbidities or frailty and diseases targeted by practice guidelines.

Pilot-testing the payoff time framework is particularly important because preventive guidelines may not be implemented in care for reasons beyond a provider's control (eg, lack of health insurance, poor adherence), or may get lost in the vast spectrum of competing demands. For example, if providers aimed to apply every preventive guideline to a typical patient panel, they would need to devote 7 additional hours each day beyond time already spent managing chronic disease and addressing acute complaints.29 Clinicians do not have any systematic method to prioritize among preventive guidelines and do not have time to implement them all.30 Therefore, linking the payoff time framework to a clinical reminder alone, without also incorporating a decision support system that facilitates information 
gathering, prioritization, and implementation, is likely to be unsuccessful. Accordingly, when we pilot-test this framework, we plan to integrate it into the electronic medical record, and to use nonphysician staff to collect information. Other important questions that need to be addressed by pilot testing include how well the payoff time performs in actual clinical practice (eg, what is the utility of this framework over usual care?), whether it slows down clinical workflow or poses an unacceptable time burden, and whether it represents good value. Piloting testing may also inform future efforts to decrease the number of data elements required.

Perhaps the most important question addressed by pilot testing may involve its acceptability for patients (eg, how to "frame" the shared decision making triggered by payoff time information31), particularly for those who prefer to continue screening even in the face of high risk or low reward. Ideally, clinical decisions should reflect a shared decision-making process between patient and physician that incorporates the individual values and priorities of a particular patient. Even if a patient's life expectancy is longer than the payoff time for a guideline, that guideline may not be advised because it does not reflect that patient's preferences. Conversely, even if a patient's life expectancy is shorter than the payoff time for a guideline, that guideline may be an appropriate topic for discussion. This discussion should not take the form of discouraging pronouncements such as "You're not going to live long enough to need this test," but rather could be introduced by statements such as "Every patient is different, and what is best for 1 person may not be best for another. Chances are that you are more likely to be harmed, or will not benefit, from this test."

It is important to note that the payoff time framework is flexible, and is not limited to colorectal cancer screening, or for that matter, preventive care practice guidelines. It is applicable to any guideline likely to result in short-term harms but longer-term benefits (eg, repair of abdominal aortic aneurysms), and therefore may eventually be applied to other practice guidelines. The payoff time framework is substantially different than other published approaches for individualizing practice guidelines because they require qualitative valuations that are often complex and are difficult to perform and apply at the point of care. $1^{-3}, 32,33$ In contrast, this framework was designed "from the ground up" to be applicable at the point of care and to inform clinical decision support systems, because of its systematic and quantitative approach.

Our modified framework still has important limitations. Although we have enhanced its generalizability and feasibility by linking it to a prognostic model not developed for any particular disease, disease-specific prognostic models may be more accurate when they are available. For example, if a patient has severe congestive heart failure, the Seattle Heart Failure model 34 may yield a more accurate estimate of life expectancy than the model of Lee et al. There will be many situations in which it is not possible to apply this framework, because it may either underestimate benefits or overestimate harms (eg,, case 3, in which that patient's particularly high risk of colorectal cancer may lead to underestimation of benefits from screening), or because factors external to the provider may limit guideline implementation (eg, lack of health insurance, lack of geographic proximity to providers, or patient-specific adherence barriers such as alcohol abuse, drug abuse, or mental health disorders). Our approach uses population-based data to inform decisions about individual patients and inferences may not always be appropriate, a caveat common to all applications of evidence to clinical decisions.

While the main limitation of this framework is its need to be validated in a clinical care setting, it has important strengths that make further study worthwhile. The framework may be a counterbalance to the blind application of guidelines that are inappropriate for individual patients, and may counter the unintended consequences of pay-for-performance 
and quality benchmarks. It could perhaps stimulate thoughtful physician-patient discussion regarding preferences for screening. If it makes valid predictions, it can be applied in a format that minimizes or eliminates the physician role in collecting information, and therefore may be compatible with emerging models for primary care such as the patientcentered medical home.35 Most importantly, application of the payoff time framework has the potential to limit inappropriate care, thereby increasing quality of care while reducing costs.

\section{Acknowledgments}

This work was funded by a Robert Wood Johnson Physician Faculty Scholar Award.

\section{REFERENCES}

1. Walter LC, Covinsky KE. Cancer screening in elderly patients: a framework for individualized decision making. JAMA. 2001; 285:2750-2756. [PubMed: 11386931]

2. Tinetti ME, Bogardus ST Jr, Agostini JV. Potential pitfalls of disease-specific guidelines for patients with multiple conditions. N Engl J Med. 2004; 351:2870-2874. [PubMed: 15625341]

3. Durso SC. Using clinical guidelines designed for older adults with diabetes mellitus and complex health status. JAMA. 2006; 295:1935-1940. [PubMed: 16639053]

4. Sultan S, Conway J, Edelman D, et al. Colorectal cancer screening in young patients with poor health and severe comorbidity. Arch Intern Med. 2006; 166:2209-2214. [PubMed: 17101938]

5. Walter LC, Bertenthal D, Lindquist K, et al. PSA screening among elderly men with limited life expectancies. JAMA. 2006; 296:2336-2342. [PubMed: 17105796]

6. Armour BS, Friedman C, Pitts MM, et al. The influence of year-end bonuses on colorectal cancer screening. Am J Manag Care. 2004; 10:617-624. [PubMed: 15515994]

7. Braithwaite RS, Concato J, Roberts MS, et al. A framework for tailoring clinical guidelines to comorbidity at the point of care. Arch Intern Med. 2007; 167:2361-2365. [PubMed: 18039996]

8. Lee SJ, Lindquist K, Segal MR, et al. Development and validation of a prognostic index for 4-year mortality in older adults. JAMA. 2006; 295:801-808. [PubMed: 16478903]

9. Beck JR, Kassirer JP, Pauker SG. A convenient approximation of life expectancy (the "DEALE"). I. Validation of the method. Am J Med. 1982; 73:883-888. [PubMed: 7148881]

10. Boyd CM, Xue Q, Simpson CF, et al. Frailty, hospitalization, and progression of disability in a cohort of disabled older women. Am J Med. 2005; 118:1225-1231. [PubMed: 16271906]

11. Ferucci L, Guralnik JM, Cavazzini C, et al. The frailty syndrome: a critical issue in geriatric oncology. Crit Rev Oncol Hematol. 2003; 46:127-137. [PubMed: 12711358]

12. Rockwood K, Mitnitski A. Frailty in relation to the accumulation of deficits. J Gerontol. 2007; 62A:722-727.

13. Nourhashemi F, Andrieu S, Gillette-Guyonnet S, et al. Instrumental activities of daily living as a potential marker of frailty: a study of 7364 community-dwelling elderly women (the EPIDOS study). J Gerontol. 2001; 56A:M448-M453.

14. Ko, CW.; Riffle, S.; Morris, C., et al. Complications after screening and surveillance colonoscopy. A-149, American Gastroenterology Association; 2007.

15. Levin TR, Zhao W, Conell C, et al. Complications of colonoscopy in an integrated health care delivery system. Ann Intern Med. 2006; 145:880-886. [PubMed: 17179057]

16. Ko CW, Sonnenberg A. Comparing risks and benefits of colorectal cancer screening in elderly patients. Gastroenterology. 2005; 129:1163-1170. [PubMed: 16230070]

17. Sturmer T, Glynn RJ, Lee IM, et al. Lifetime cigarette smoking and colorectal cancer incidence in the Physicians' Health Study I. J Natl Cancer Inst. 2000; 92:1178. [PubMed: 10904092]

18. Martinez ME, Giovannucci E, Spiegelman D, et al. Leisure-time physical activity, body size, and colon cancer in women. Nurses' Health Study Research Group. J Natl Cancer Inst. 1997; 89:948. [PubMed: 9214674] 
19. Giovannucci E, Ascherio A, Rimm EB, et al. Physical activity obesity, and risk for colon cancer and adenoma in men. Ann Intern Med. 1995; 122:327. [PubMed: 7847643]

20. Cho E, Smith-Warner SA, Ritz J, et al. Alcohol intake and colorectal cancer: a pooled analysis of 8 cohort studies. Ann Intern Med. 2004; 140:603. [PubMed: 15096331]

21. Larsson SC, Orsini N, Wolk A. Diabetes mellitus and risk of colorectal cancer: a meta-analysis. J Natl Cancer Inst. 2005; 97:1679. [PubMed: 16288121]

22. Dube C, Rostorn A, Lewin G, et al. US Preventive Services Task Force. The use of aspirin for primary prevention of colorectal cancer: a systematic review prepared for the US Preventive Services Task Force. Ann Intern Med. 2007; 146:365-375. [PubMed: 17339622]

23. Chlebowski RT, Wactawski-Wende J, Ritenbaugh C, et al. Estrogen plus progestin and colorectal cancer in postmenopausal women. N Engl J Med. 2004; 350:991. [PubMed: 14999111]

24. Johns LE, Houlston RS. A systematic review and meta-analysis of familial colorectal cancer risk. Am J Gastroenterol. 2001; 96:2992-3003. [PubMed: 11693338]

25. Weinberg DS. In the clinic: colorectal cancer screening. Ann Intern Med. 2008 ITC2-1-16.

26. Baxter NN, Tepper JE, Durham SB, et al. Increased risk of rectal cancer after prostate radiation: a population-based study. Gastroenterology. 2005; 128:819. [PubMed: 15825064]

27. Hodgson DC, Gilbert ES, Dores GM, et al. Long-term solid cancer risk among 5-year survivors of Hodgkin's lymphoma. J Clin Oncol. 2007; 25:1489. [PubMed: 17372278]

28. Delhougne B, Deneux C, Abs R, et al. The prevalence of colonic polyps in acromegaly: a colonoscopic and pathological study in 103 patients. J Clin Endocrinol Metab. 1995; 80:3223. [PubMed: 7593429]

29. Yarnall KSH, Pollak KI, Ostbye T, et al. Primary Care: is there enough time for prevention? Am J Public Health. 2003; 93:635-641. [PubMed: 12660210]

30. Dalal M, Bradley E, Braithwaite RS. Prioritizing clinical practice guidelines in the primary care setting. Med Decis Making. 2008; 28:150-155. E12.

31. Salovey, P.; Wegener, DT. Communicating about health: message framing, persuasion, and health behavior. In: Suls, J.; Wallston, KA., editors. Social Psychological Foundations of Health and Illness. Malden, MA: Blackwell Publishing; 2003.

32. Holmes HM, Hayley DC, Alexander GC, et al. Reconsidering medication appropriateness for patients late in life. Arch Intern Med. 2006; 166:605-609. [PubMed: 16567597]

33. Boyd CM, Darer J, Boult C, et al. Clinical practice guidelines and quality of care for older patients with multiple comorbid diseases: implications for pay for performance. JAMA. 2005; 294:716724. [PubMed: 16091574]

34. Levy WC, Mozaffarian D, Linker DT, et al. The Seattle heart failure model: prediction of survival in heart failure. Circulation. 2006; 113:1424-1433. [PubMed: 16534009]

35. The Advanced Medical Home: A Patient-Centered, Physician-Guided Model of Health Care American College of Physicians: A Policy Monograph. 2006 [Accessed 11/05/08]. Available at: http://www.acponline.org/advocacy/where_we_stand/policy/adv_med.pdf. 


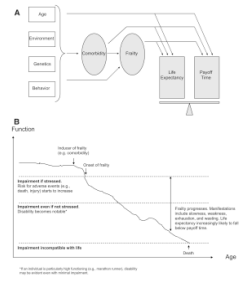

FIGURE 1.

Conceptual model of how comorbidity and frailty impact life expectancy and a guideline's payoff time in modified framework. A, Comorbidity and frailty not only impact life expectancy but also may impact the payoff time of a guideline (earliest time when cumulative incremental benefits first exceed cumulative incremental harms). If the life expectancy falls below the payoff time, then the practice guideline is more likely to result in harm than benefit. Frailty may or may not arise from a comorbidity, and even if it does arise from a comorbidity, it may impart an incremental effect on life expectancy distinct from that of the comorbidity. B, Schematic diagram of frailty trajectory in hypothetical patient. As frailty worsens, it increases vulnerability to death, thereby lowering life expectancy and increasing the likelihood that life expectancy will fall below any particular payoff time. 


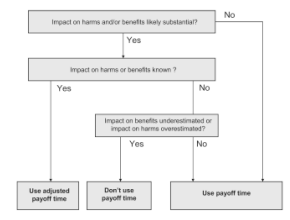

FIGURE 2 .

Schematic of decision rules for applying payoff 


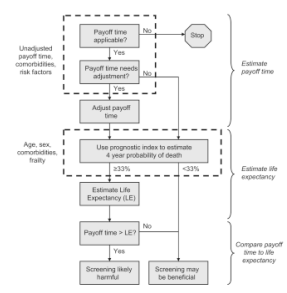

FIGURE 3.

General schematic of how payoff time may inform clinical decision making. 


\section{TABLE 1}

Prognostic Index of Lee et al. Of the 13 Data Elements That are Required for This Index, as Many as 8 may be Obtained From an Electronic Medical Record System, Leaving as few as 5 Items for Patient Survey

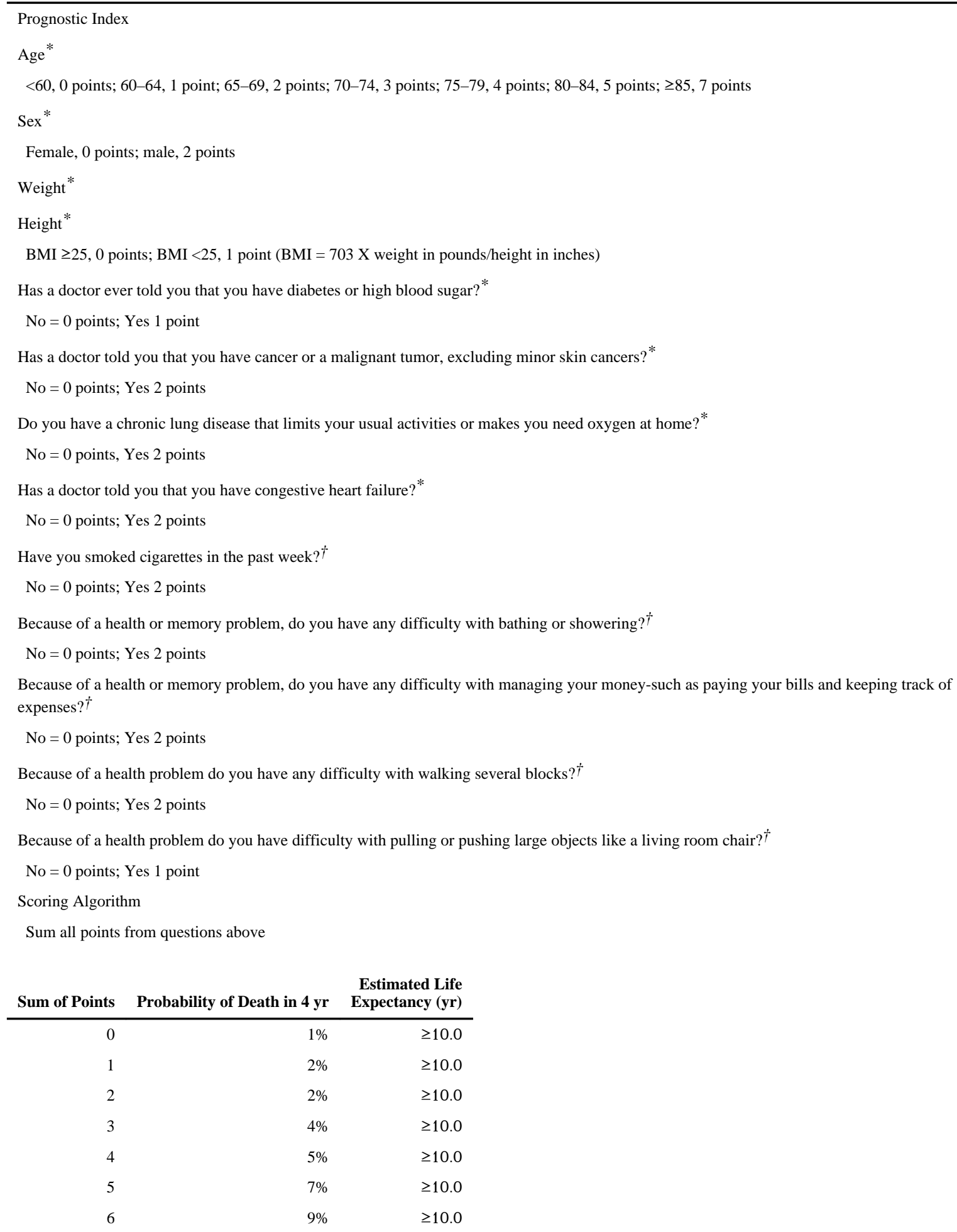


Estimated Life

\begin{tabular}{rrr} 
Sum of Points & Probability of Death in $\mathbf{4} \mathbf{y r}$ & Expectancy (yr) \\
\hline 7 & $14 \%$ & $\geq 10.0$ \\
8 & $20 \%$ & $\geq 10.0$ \\
9 & $22 \%$ & $\geq 10.0$ \\
10 & $28 \%$ & $\geq 10.0$ \\
11 & $44 \%$ & 6.9 \\
12 & $46 \%$ & 6.5 \\
13 & $57 \%$ & 4.7 \\
$\geq 14$ & $66 \%$ & $\leq 3.7$
\end{tabular}

Data element may be obtained from survey at check in and/or electronic medical record system.

${ }^{\dagger}$ Data element may be obtained from survey at check-in. 
TABLE 2

Adjustment of Payoff Time Based on Individual Characteristics*

\begin{tabular}{|c|c|c|c|}
\hline Characteristic & $\begin{array}{l}\text { Relative Risk (RR) for } \\
\text { Colorectal Cancer } \\
\text { Deaths (Amplifies Potential } \\
\text { Benefits) }\end{array}$ & $\begin{array}{l}\text { Relative Risk (RR) for } \\
\text { Colonoscopy } \\
\text { Deaths (Amplifies } \\
\text { Potential Harms) }\end{array}$ & $\begin{array}{c}\text { Can Use } \\
\text { Adjusted } \\
\text { Payoff Time? }\end{array}$ \\
\hline Smoking $17^{\dagger+}$ & 1.8 & Unknown $\S$ & Yes \\
\hline Obesity $18,19^{\dagger}$ & 1.5 & Unknown $\S$ & Yes \\
\hline Heavy alcohol 20 II & 1.3 & Unknown $\S$ & Yes \\
\hline Diabetes $21^{\dagger} \mathscr{I}$ & 1.3 & Unknown $\S$ & Yes \\
\hline Aspirin (regular use) $22 \%$ & 0.8 & Unknown $\S$ & Yes \\
\hline NSAID (regular use) $22 \mathscr{I}$ & 0.7 & Unknown $\S$ & Yes \\
\hline Hormone replacement therapy 23 I & $0.6^{\prime \prime}$ & Unknown $\S$ & Yes \\
\hline Coumadin $14 \pi$ & Unknown $^{* *}$ & 4.0 & Yes \\
\hline $\begin{array}{l}\text { American Anesthesiology Society Class } 1 \text { ("normal } \\
\text { healthy patient") } 14^{\mathscr{I}}\end{array}$ & Unknown $^{* *}$ & 0.7 & Yes \\
\hline $\begin{array}{l}\text { American Anesthesiology Society Class } 3 \text { ("severe } \\
\text { systemic disease") } 14 \pi \text { I }\end{array}$ & Unknown $^{* *}$ & 4.3 & Yes \\
\hline 1st degree relative with CRC, age unknown24 & 2.3 & Unknown $\S$ & Yes \\
\hline 1st degree relative with CRC, age $<4524^{*}$ & 3.9 & Unknown $\S$ & Yes \\
\hline$>1$ st degree relative with $\mathrm{CRC} 24^{\neq}$ & 4.3 & Unknown $^{\S}$ & Yes \\
\hline Inflammatory bowel disease 25 II & Variable; $>>1^{\dagger \dagger}$ & Unknown $\S$ & No \\
\hline $\begin{array}{l}\text { Personal history of colon cancer or adenomatous } \\
\text { polyps } 24 \pi\end{array}$ & Variable; $>>1^{\dagger \dagger}$ & Unknown $\S$ & No \\
\hline History of pelvic radiation $26^{\pi /}$ & Unknown but likely to be $>1^{\dagger \dagger}$ & Unknown $\S$ & No \\
\hline Hodgkins Lymphoma27 7 I & Unknown but likely to be $>1^{\dagger \dagger}$ & Unknown $\S$ & No \\
\hline Acromegaly $28 \pi$ & Unknown but likely to be $>1^{\dagger \dagger}$ & Unknown $\S$ & No \\
\hline \multicolumn{4}{|l|}{$\begin{array}{l}* \\
\text { These characteristics were selected because publishe } \\
\text { multiple studies were performed) were consistent in } m \\
\text { with the prognostic index, and an additional } 12 \text { may be } \\
\text { patient survey. }\end{array}$} \\
\hline \multicolumn{4}{|l|}{${ }^{\dagger}$ Data already obtained to estimate payoff time. } \\
\hline \multicolumn{4}{|l|}{ Data may be obtained from survey at check-in. } \\
\hline \multicolumn{4}{|c|}{$\begin{array}{l}\S \text { There is no a priori reason to expect that the RR is substantially lower than } 1 \text { (in which case, the impact on harms may be overestimated), } \\
\text { therefore it remains valid to use the adjusted payoff time. Note that if there were an a priori reason to expect that the RR may be substantially lowe } \\
\text { than } 1 \text { (in which case, the impact on harms may be overestimated), it would not be valid to use the adjusted payoff time. }\end{array}$} \\
\hline \multicolumn{4}{|c|}{ II Data may be obtained from electronic medical record or from survey at check-in. } \\
\hline Based on hazard ratio. & & & \\
\hline
\end{tabular}


${ }^{\dagger \dagger}$ There is an a priori reason to expect that the RR may be substantially greater than 1 (in which case, the impact on benefits may be underestimated), therefore it is not valid to use the adjusted payoff time. 


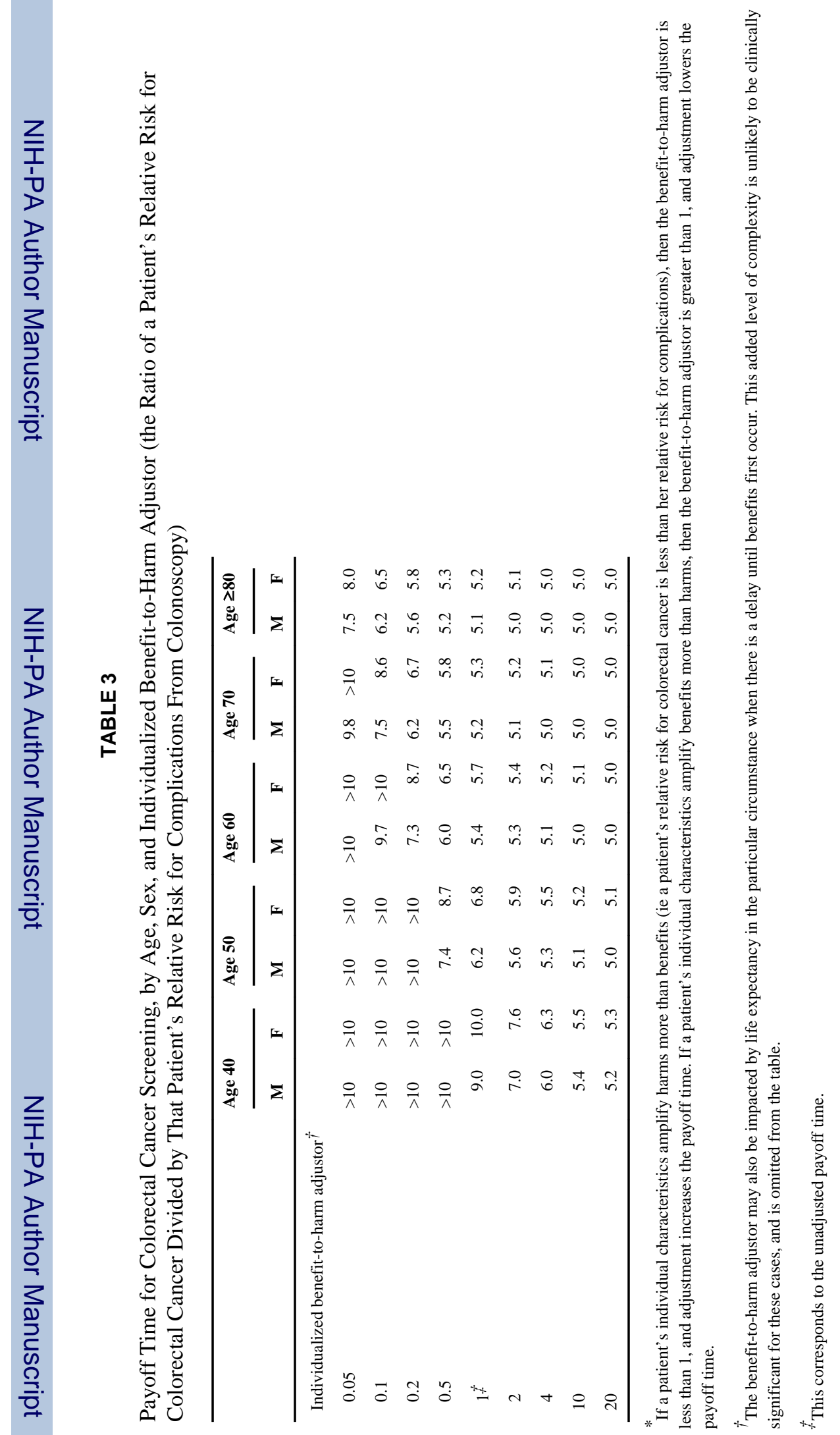

Med Care. Author manuscript; available in PMC 2011 April 15. 